\title{
Zona de promesas. Primera generación de estudiantado universitario de la Universidad Nacional de La Plata, Argentina (2008-2015)
}

\author{
A Promise Zone. First Generation of University Students of the National University of La \\ Plata, Argentina (2008-2015)
}

\section{Zona de promessas. Primeira geração de estudantes universitários da Universidade Nacional de La Plata, Argentina (2008-2015)}

Resumen: Aquíse propone reflexionar en torno de las políticas inclusivas en materia de comunicación/ educación implementadas en los últimos años en la Argentina. En esta oportunidad, se trabajará con datos cuantitativos sobre estudiantes ingresantes de todas las facultades de la Universidad Nacional de La Plata entre 2008-2015. Esta universidad nacional es de carácter público, gratuito y de ingreso irrestricto. La información fue relevada a partir de los datos que proporcionó el Centro Superior para el Procesamiento de la Información (CeSPI) de la UNLP. El recorte temporal de los años 2008 a 2015 obedece a dos propósitos. El primero se vincula con que, en 2008, se comienza a cumplir con la obligatoriedad de la educación secundaria en el ámbito nacional, a partir de la Ley 26606 (2006), y en la provincia de Buenos Aires, con la sanción de la Ley de Educación Provincial n. ${ }^{\circ} 13688$ del 2007. El segundo indica que también en 2008 se lanza el Plan de Finalización de Estudios Primarios y Secundarios (FinES) como una iniciativa estatal para cumplir con lo señalado en la obligatoriedad de la educación secundaria consignada en la legislación vigente.

Palabras claves: Comunicación; educación; política pública; universidad; derechos. 
http://doi.org/10.15359/ree.24-3.27

http://www.una.ac.cr/educare

educare@una.ac.cr

\begin{abstract}
We propose to reflect on inclusive policies on communication/education implemented in the last years in Argentina. We will work with quantitative data about students admitted to all faculties of the Universidad Nacional de La Plata (UNLP) between 2008-2015. This national university is public, free, and implements unrestricted admission policies. Information was collected from data provided by the Centro Superior para el Procesamiento de la Información (CeSPI, Superior Center for Information Processing) of the UNLP. Two reasons justify the selection of the 2008-2015 period. The first one is linked to Law 26,606 (2006) on compulsory secondary education that came into effect in 2008 at the national level, and in the Buenos Aires province, with the approval of Provincial Education Law No. 13,688 (2007). The second reason relates to the Plan for Primary and Secondary Education Completion (FinES), launched in 2008, as a state initiative to comply with the current legislation about the obligatoriness of the secondary education.
\end{abstract}

Keywords: Communication; education; public policy; university; rights.

Resumo: A proposta desse trabalho é reflexionar sobre as políticas inclusivas em matéria de comunicação/educação implementadas nos últimos anos da Argentina. Nesta oportunidad, se trabajará com dados cuantitativos de estudantes ingressantes de todas as faculdades da Universidade Nacional de La Plata desde 2008-2015. Esta universidade é de caráter público, gratuito e de acesso irrestrito. A informacao foi consultada a partir dos dados proporcionados pelo Centro Superior para o Processo de Informação (CeSPI) da UNLP. O período temporal dos anos 2008 a 2015 responde a dos propósitos. O primeiro está vinculado a que, em 2008, começa a ser obrigatória a educação secundária no âmbito nacional, a partir da Lei 26.606 (2006), e na província de Buenos Aires, com a sanção da Lei de Educação Provincial N 13.688 de 2007. O segundo indica que também em 2008 é lançado o Plano de Finalização de Estudos Primários e Secundários (Fines), como uma medida estatal para cumprir com o indicado sobre ser obrigatório a educação secundária consignada na lei vigente.

Palavras-chave: Comunicação; educação; política pública; universidade; dereitos.

\title{
Introducción
}

Una de las intenciones de este artículo consiste en reflexionar acerca de los alcances y las limitaciones de las políticas inclusivas en materia de comunicación/educación en los últimos años de la Argentina. Las perspectivas críticas que sostenemos de comunicación/educación se han articulado con políticas públicas de inclusión educativa al considerar a la comunicación y la educación como derechos humanos.

De manera específica, en esta oportunidad trabajaremos acerca de algunos datos cuantitativos de estudiantado universitario ingresante de las facultades de la Universidad Nacional de La Plata (UNLP), desde 2008 hasta 2015. La información fue relevada a partir de los datos proporcionados por el Centro Superior para el Procesamiento de la Información (CeSPI) de la UNLP. Este organismo institucional se encarga de centralizar todas las informaciones que le remiten las dependencias de la universidad. La información remitida se corresponde con los datos que manejan las 18 facultades y la Escuela Universitaria de Recursos Humanos del Equipo de Salud (EURHES). El listado completo de las facultades es el siguiente: Facultad de Arquitectura yUrbanismo 
(FAyU); Facultad de Bellas Artes (FBA); Facultad de Ciencias Agrarias y Forestales (FCAyF); Facultad de Ciencias Astronómicas y Geofísicas (FCAyG); Facultad de Ciencias Económicas (FCE); Facultad de Ciencias Exactas (FCEx); Facultad de Ciencias Jurídicas y Sociales (FCJyS); Facultad de Ciencias Médicas (FCM); Facultad de Ciencias Naturales y Museo (FCNyM); Facultad de Ciencias Veterinarias (FCV); Facultad de Humanidades y Ciencias de la Educación (FHyCE); Facultad de Informática (FInf); Facultad de Ingeniería (FI); Facultad de Odontología (FO); Facultad de Periodismo y Comunicación Social (FPyCS); Facultad de Psicología (FP); Facultad de Trabajo Social (FTS). En 2010 se fundó la Facultad de Ingeniería e Informática (Flel), que dicta la carrera de Ingeniería en Computación, gestionada de manera conjunta por la FI y la FInf. Cabe destacar que el CeSPI está encargado de procesar toda la información que le reportan las secretarías académicas de todas las facultades y, por lo tanto, es la fuente de estadísticas oficiales que posee la UNLP para reportar a la Secretaría de Políticas Universitarias del Ministerio de Educación de la Nación.

El recorte temporal de los años 2008 a 2015 obedece a dos propósitos. El primero se corresponde porque en 2008 se comienza a cumplir con la obligatoriedad de la educación secundaria en el ámbito nacional, a partir de la Ley 26606 del 2006, y en la provincia de Buenos Aires, con la sanción de la Ley de Educación Provincial n. 13.688 del 2007. El segundo indica que también en 2008 se lanza el Plan de Finalización de Estudios Primarios y Secundarios (FinES), como una iniciativa estatal para cumplir con lo señalado en la obligatoriedad de la educación secundaria consignada en la legislación vigente. El programa FinEs consistía en lo siguiente:

En consonancia con la extensión de la escolaridad obligatoria hasta la finalización de la educación secundaria, se inició en el año 2008 el "Plan de Finalización de Estudios Primarios y Secundarios" (FinEs) con alcance nacional. La primera etapa de este plan priorizó a jóvenes de 18 a 25 años que terminaron de cursar el último año del nivel medio como estudiantes regulares y adeudan materias y en una segunda etapa, a las personas adultas mayores de 25 años en la misma condición. Incluye instancias presenciales, semipresenciales y a distancia (de la Fare, 2010, p. 40).

El corte finaliza en el 2015 porque era la información pública que disponía el CeSPI cuando solicitamos los datos para la realización de nuestra investigación, aunque logró brindar información de 2016 ante una de nuestras preguntas. De todas maneras, creemos que se trata de un panorama que puede dar cuenta de una clara tendencia de los ingresos en la UNLP en un periodo de tiempo significativo.

La solicitud de información que realizamos se inscribe en un cambio de gobierno en el ámbito nacional. La asunción del presidente Mauricio Macri representa una clara reorientación de las políticas públicas y de las estrategias macroeconómicas, que ya se expresan en devaluación de la moneda nacional, aumento de las tarifas de servicios públicos, endeudamiento externo, transferencia de recursos de los sectores más pobres hacia los más concentrados. Para más especificidad, desde la asunción del gobierno macrista el régimen económico valora la 
http://doi.org/10.15359/ree.24-3.27

http://www.una.ac.cr/educare

educare@una.ac.cr

especulación financiera como eje del proceso de acumulación; solo en los primeros 10 meses de 2016 las colocaciones de deuda acumularon USD 52.000 millones. "La rentabilidad esperada de las opciones de inversión productiva [resultó] escasa como consecuencia de la recesión interna, la apertura comercial, la agudización de la apreciación cambiaria y las escasas o nulas políticas industriales" (Arceo et al., 2016, p. 1).

Estas menciones a la modificación de la orientación de política económica ilustran un panorama totalmente diferente al que se pretendió instalar en el ciclo del gobierno kirchnerista. En ese entonces se produjo un desendeudamiento externo y no alineamiento con los Estados Unidos:

El control de la salida de dólares, la requisa a las cuevas de los especuladores financieros, la difusión pública de algunos nombres de los operadores involucrados en operativos desestabilizadores como fue el caso del presidente de la Shell, los precios cuidados, la utilización de las reservas del Banco Central y los swaps con China como recurso para sostener la pulseada por el tipo de cambio (Basualdo et al., 2015, p. 4).

Estas advertencias centradas en aspectos estructurales evitan caer en explicaciones deterministas de lo económico, como una única fuente de argumentación de las problemáticas sociales. Más bien se pretende indicar un claro direccionamiento de ciertas variables marcadoras de un horizonte que pretende lograr la elite gobernante de la Argentina. La historia reciente nos muestra que los recursos destinados al pago de la deuda y a cubrir el déficit de la fuga de divisas se quitan de los fondos destinados a políticas públicas inclusivas.

La propuesta de este texto consiste en sobrevolar algunas de las cuestiones estructurales de la sociedad argentina, para luego dar paso a una caracterización de la situación de la educación universitaria y así ingresar en el caso específico de estudiantes ingresantes de la UNLP durante el periodo 2008-2015.

\section{¿Una sociedad más igualitaria?}

América Latina es la región más desigual del mundo y eso se sustenta en la interrelación de dinámicas de expropiación y apropiación por las cuales una parte toma recursos y se los excluye a otros (Reygadas, 2008). Si los esfuerzos gubernamentales se centran en favorecer la fuga de capitales y el pago a fondos de especulación internacionales, es evidente que esos recursos serán expropiados a otros sectores sociales que no hacen de la especulación financiera su rutina de trabajo. Además de estas dinámicas en tensión, también entran en disputa las interpretaciones acerca de cómo entender las causalidades del interjuego entre las expropiaciones y las apropiaciones de recursos.

Quizás el trabajo más relevante que se inscribe en la tónica del análisis de la desigualdad en la Argentina sea el realizado por Kessler (2014), quien toma como recorte temporal la década 2003-2013. Afirma que la desigualdad permitió reinscribir a la pobreza dentro de la dinámica social 
http://doi.org/10.15359/ree.24-3.27

y entenderla como un subproducto de las inequidades. Así conjetura que "hubo ... movimientos hacia una mayor igualdad en ciertas dimensiones, pero también la perdurabilidad, o el reforzamiento en ciertos casos, ... de desigualdades en otras"(Kessler, 2014, p. 14). Para desandar tal propósito, sobre la base de los criterios de relevancia, dinámicas propias y controversia, estableció un conjunto de dimensiones analíticas: distribución del ingreso; educación, salud y vivienda; territorios, infraestructura y cuestión rural; inseguridad y delito. La articulación de Kessler conjuga elementos donde enfatiza en lo controversial de ciertas esferas que fueron descartadas del análisis porque su nivel de controversias es menor. Enumera las iniciativas de diversidad sexual, la ley de matrimonio igualitario (Ley . $^{\circ} 26.618$ ) y la ley migratoria (Ley $n .{ }^{\circ} 25.871$ ) que representan un grado importante de aceptación social en las evaluaciones realizadas por los actores sociales y por los indicadores relevados. Consigna que no todos los grupos sociales han tenido el mismo trato, como es el caso de los pueblos originarios y que, en el caso del género -mujeres-, las inequidades siguen presente en sus ingresos y mercado de trabajo.

Un aspecto omitido en este estudio, y en otro del que recuperaremos algunas cuestiones (Kessler, 2016), radica en que la Ley de Servicios de Comunicación Audiovisual n. 26.552 no aparece siquiera mencionada como una iniciativa destinada a cuestionar la desigualdad. Para plantearlo en términos de pregunta: ¿se puede analizar la estructura de la sociedad argentina sin interrogarse, al menos tangencialmente, por la conformación del sistema de medios de comunicación? Solo queremos señalar la omisión porque cada vez más las sociedades capitalistas posindustriales tienden a estructurarse a partir de la presencia de los medios de comunicación. Además, si lo inscribimos dentro de la organización argumental sugerida por Kessler, el nivel de controversia que suscitó la Ley de Servicios de Comunicación Audiovisual se figuró como un parte aguas de la segunda etapa de la década que analiza. Ni hablar que trascendió hasta la finalización del kirchnerismo y que los artículos que prohibían la concentración de frecuencias y paquetes accionarios de medios fueron modificados por decretos de necesidad y urgencia firmados por el presidente Macri. Los grandes medios de comunicación, en sus directorios, tienen representantes claros de la oligarquía diversificada (Basualdo, 2005) de nuestro país; por ejemplo, el diario Clarín llegó a cotizar en 2008 en la Bolsa de Valores de Londres (Sivak, 2013). ¿La preeminencia de monopolios mediáticos contribuye al sostenimiento de esferas sociales más desiguales? Solo anhelamos instalar una dimensión de análisis que problematice la desigualdad en nuestras sociedades latinoamericanas.

La cuestión de la distribución del ingreso es clave para atender las características del período analizado. Hubo un contexto de recuperación del empleo, entre 2003 y 2009, donde se crearon 4,9 millones de puestos de trabajo; la tasa de desempleo pasó del 21,5\% en 2002, se redujo a $7,9 \%$ en el segundo trimestre de 2010. Esto, junto con la revitalización del valor institucional del salario mínimo para mejorar la distribución y el incremento del trabajo registrado, fueron las condiciones para alcanzar un efecto directo de la disminución de la desigualdad (Kessler, 2014). La profundización de una desigualdad de ingresos tuvo una rápida reversión en ese sentido y la recuperación posterior quizá sea menos estable. La incorporación del polo marginal de del sector trabajador es una de las cuestiones que todavía se encuentran sin atender (Kessler, 2014). 
http://doi.org/10.15359/ree.24-3.27

http://www.una.ac.cr/educare

educare@una.ac.cr

Para entrar en una de las dimensiones específicas de nuestro trabajo, en educación los debates han sido constantes. La temática siempre aparece en discusiones de campaña, conversaciones cotidianas, en discursos mediáticos. En el fragor de esas discusiones, podemos sostener que a la educación se le pide que resuelva los problemas que ni la política, ni la economía lograron solucionar para todo el conjunto de la sociedad. Así, el umbral de expectativas de la que la escuela es la depositaria excede por completo el alcance de sus posibilidades. Hecha esta advertencia, en los últimos años nuestro país ha mejorado notablemente la tasa de escolarización en todos niveles, como así también se extendió la obligatoriedad con la sanción de la Ley de Educación Nacional n. ${ }^{\circ}$ 26 606. Las estadísticas del Sistema de Información de Tendencias Educativas de América Latina (SITEAL), dependiente de la Unesco, señalan un aumento de la tasa de escolarización de todos los sectores sociales de la Argentina en el periodo 2000-2016 (Unesco, 2017).

La obligatoriedad se extendió desde los 5 años hasta la finalización de la educación secundaria. Entre otro conjunto de medidas, se estableció la Ley de Financiamiento Educativo que establecía el $6 \%$ del PBI para educación, el Programa Conectar lgualdad, la creación mediante la ley nacional- del Instituto de Formación Docente (Infod), entre varias más. Ese aumento exponencial de la matrícula permitió el ingreso, de sectores sociales históricamente postergados, a las instituciones educativas. Las instituciones y sus actores se enfrentaron a nuevos escenarios que alteraron ciertas prácticas históricamente sedimentadas para clases sociales que tenían un vínculo más extendido con la escuela.

El nivel de inversión estructural casi no ha sido cuestionado. Sí existen discusiones potentes acerca de la calidad educativa, la perdurabilidad de la desigualdad dentro del mismo sistema educativo y los mecanismos de evaluación o la construcción de indicadores confiables para diagnosticar estas problemáticas en la Argentina. Uno de los indicadores más extendido es el Programa para la Evaluación Internacional de los Estudiantes (PISA), creado por la Organización para la Cooperación y el Desarrollo Económico (OCDE). Algunas posiciones señalan que "lo cierto es que el PISA es uno de los indicadores sistemáticos con el que contamos para comparar los desempeños de nuestro país respecto de otros, y que en él nos va mal" (Kessler, 2014, p. 130). Mientras que otras afirman que PISA logró una hazaña ideológica "[al] imponer como evidente y necesaria la premisa de que los sistemas escolares de todos los países pueden ser evaluados mediante la aplicación de una misma prueba aplicada a un conjunto de estudiantes elegidos al azar" (Gentili, 2015, p. 152). Los intereses que subyacen a las pruebas PISA guardan una íntima relación con el éxito económico, además establece la posibilidad de la creación de un ranking de escuelas. La pregunta que guía esta iniciativa, cuya implementación se remonta al año 2000, es la siguiente: ¿qué es importante que la ciudadanía sepa y sea capaz de hacer? A simple vista, esta respuesta sería infructuosa de responderla con "la aplicación de una prueba trienal de dos horas de duración" (Gentili, 2015, p. 165). La evaluación no es el aspecto que se cuestiona, sino el mecanismo de realizar esta práctica a partir de intereses supuestamente neutrales y universales. Esta discusión aún no encontró posiciones superadoras, pero sí es un terreno fértil 
para operaciones mediáticas, con argumentos simplistas, destinados a atacar al colectivo de docentes y la educación pública.

La cuestión de la ampliación del ingreso a todos los ámbitos institucionales educativos, que se expresa en los aumentos de las matrículas de los más variados sectores sociales, es un aspecto para caracterizar positivamente a este último periodo. Ciertas tendencias de análisis, que logran un punto de fijación muy sólido en el sentido común, sugieren que esto se trata de un acontecimiento vacío que podría sintetizarse del siguiente modo: entran todos, pero ninguno aprende. Con el imperativo que defendemos de que el paso por los ámbitos institucionales educativos debe promover nuevos saberes para la lectura y la reescritura del mundo -es decir jamás desconocer la importancia de los aprendizajes, las evaluaciones y la finalización acreditada de las trayectorias- nuestra posición es un tanto diferente. Para que se haya logrado el ingreso tan sostenido en el tiempo y fundamentalmente de los sectores más bajos es porque el Estado generó una trama de políticas para que accedieran a territorios que suponían vedados para ellos. Benza (2016) afirma:

La recuperación de los ingresos que se inició en 2003 involucró a todas las clases, pero quienes se vieron más favorecidas fueron las clases populares: sus ingresos aumentaron alrededor de $77 \%$ entre 2003 y 2010. Las clases medias, en conjunto, se vieron menos beneficiadas (el crecimiento de sus ingresos fue de $37 \%$ ), pero en ellas hubo cierta progresividad en la distribución en tanto los mayores incrementos que ocurrieron en los grupos de menor nivel. Por último, las clases altas habrían tenido mejoras aún menores, aunque que hay que tomar con recaudos por las limitaciones de los datos que utilizamos. (pp. 127-128)

Los estudios sociológicos respecto de las clases altas coinciden en la subinformación de sus ingresos a los que apelan constantemente quienes integran las clases altas de nuestro país (Gessaghi, 2016; Heredia, 2016). Nuestras investigaciones sostienen que el ingreso de una subjetividad a una institución educativa ensancha su campo de experiencias y también que el máximo nivel educativo que obtiene una generación es el mínimo que le va a exigir a la siguiente (Martínez, 2015; Morabes et al., 2017).

\section{La cuestión universidad}

Una vez efectuada la breve caracterización de los años recientes, con especial énfasis en el corte 2003-2015, iniciaremos una aproximación a contextualizar nuestros interrogantes del comienzo. Para estos efectos queremos agregar el dato que surge del censo nacional de 2010, que señala que la población más numerosa de la Argentina se aloja en la franja etaria que va de los 15 a los 19 años. Los factores para que ello ocurra se deben, principalmente, al descenso de la mortalidad infantil y al leve retraso de la tasa general de fecundidad. Es decir, de 2001 a 2010 disminuye el número de menores de 15 años. De acuerdo con la información del último censo, 
http://doi.org/10.15359/ree.24-3.27

http://www.una.ac.cr/educare

educare@una.ac.cr

la población argentina experimenta un crecimiento relativamente lento, hay un proceso de envejecimiento demográfico: aumentan las personas de edad más avanzada que las menores.

El sistema universitario argentino tiene la mayor cobertura de la matrícula de América Latina, porque tiene una tasa bruta de $38 \%$ y una tasa neta de $20 \%$ que abarca a la población entre los 18 y los 24 años (Secretaría de Políticas Universitarias del Ministerio de Educación de la Nación, 2011). También la Argentina es uno de los países con mayor participación de personas provenientes del quintil más pobre del $20 \%$, del 2001 hasta 2011 tuvo un incremento de su matrícula del $22 \%$, gracias a la expansión institucional y la creación de diez universidades nacionales en todo el país (Ruta, 2015). En 1992 había 38 universidades y en 2014 se llegó a 54 en todo el territorio nacional, con una importante localización en distritos del Conurbano Bonaerense, que tenían una clara postergación en materia de demandas educativas insatisfechas. Ruta (2015) afirma que eso provocó "el incremento de la matrícula universitaria (nuevos inscriptos y reinscriptos) de la 'primera generación de estudiantes universitarios', con una fuerte presencia en las universidades nacionales del Área Metropolitana de Buenos Aires (pp. 321-322).

A partir de lo anterior, se complejizan los escenarios institucionales y la cuestión de la inclusión se torna en un elemento crucial. Sobre todo porque se presenta una heterogeneidad social y diversidad cultural donde la desigualdad distribución del capital económico, cultural y escolar (Bourdieu, 2005) se convierte en un condicionante de la finalización exitosa de una trayectoria educativa. Ruta (2015) agrega el concepto de inclusión universitaria que implica superar el acceso formal de estudiantes a las instituciones.

De este modo, se plantea como meta la igualdad de los resultados a través de la generación de condiciones que garanticen y promuevan la permanencia y la graduación de los nuevos actores, operando sobre los efectos de la desigualdad social y salvaguardando los derechos de los grupos excluidos del sistema por causas económicas, culturales o étnicas. (p. 323)

En esta dirección, una política de becas estudiantiles es un elemento clave para la inclusión universitaria, pero en los últimos tiempos también se combinó con el fomento de programas de financiamiento para infraestructura, investigación y aumentos salariales para el personal docente y no docente.

La masificación de la matrícula universitaria produjo un cúmulo de estudios destinados a indagar sobre el acceso, la permanencia y la graduación de estudiantes. Por ejemplo, Ezcurra (2011) menciona que para ciertos sectores sociales la experiencia universitaria se transforma en una inclusión excluyente, por las diferencias que existen entre sus experiencias escolares y a las que los enfrenta el nuevo ámbito universitario. Donde, en ocasiones, se ven obligados a reproducir contenidos que poco les significan, pero a lo que responden aumentando sus posibilidades de apropiación y lo convierten en su emblema cuando lo logran. Colabella y Vargas (2014) realizan un análisis de las condiciones de acceso, permanencia y aprendizaje del oficio 
de ser estudiantado universitario en la Universidad Nacional Arturo Jauretche, localizada en el partido de Florencio Varela. Lo interesante es que no lo construyen a partir del análisis exclusivo de su transitar por las diversas facultades, sino que también profundizan el impacto que esta nueva experiencia les suscita a estudiantes en su relación con sus entornos inmediatos. Ambos estudios coinciden en señalar la relatividad de la democratización del acceso a la educación universitaria, al mismo tiempo que señalan que esta discusión se convertirá en un factor crucial para los próximos debates de la educación superior.

Surge así un falso dilema que se actualiza entre la universalización y la calidad. Dilema que adquiere espesor en campañas políticas coyunturales y en discursos cristalizados del sistema concentrado de medios de comunicación, que expande esta tensión como una verdad revelada a todo el tejido social. Si bien no es una característica que atañe solo a denigrar al sistema universitario, más bien se viraliza hacia todo el sistema público de enseñanza. Se corporiza en un núcleo duro del sentido común que horada el sentido estratégico de uno de los mayores capitales simbólico que consiguió nuestro país respecto de otros. El crecimiento de las universidades argentina es sostenido; pero en el caso de las universidades de gestión privada ha sido mayor, tanto en la matricula como en la graduación (Secretaría de Políticas Universitarias del Ministerio de Educación de la Nación, 2011).

Este activo de la sociedad argentina, nos referimos al sistema universitario en términos amplios, también evidencia zonas problemáticas a las cuales atender. En todos los quintiles, el porcentaje alcanzado de ingreso y de graduación, la Argentina tiene un promedio mayor que el resto de los países latinoamericanos. Sin embargo, cuando se discriminan esas cifras se comprueba que para nuestro país el mayor grado de acceso y graduación acontece en los sectores altos (Aberbuj y Zacarías, 2015). Circunstancia que también se replica para todo el continente, solo que con un mayor número absoluto para el resto de los países en relación con el nuestro, y refuerza la condición de desigualdad de Latinoamérica.

En la Argentina, la eliminación de los aranceles y de los exámenes de admisión se dio con el peronismo, hecho que generó una fuerte expansión histórica de la matrícula. Aberbuj y Zacarías (2015) señalan: "A sabiendas que el nivel socioeconómico es la variable que mejor predice el éxito académico, el trato igualitario a quienes son originariamente desiguales refuerza las inequidades preexistentes" (p. 368). De allí que afirmen que el mayor grado de abandono en las universidades se produzca durante el ingreso y el primer año de la carrera. Se conectan el enfrentamiento con nuevas lógicas institucionales, como así también de las tensiones que se presentan entre la enseñanza y el oficio de estudiante, que no surge espontáneamente, sino que requiere de procesos específicos de formación. Además, nos atrevemos a enunciar que es necesario problematizar cuál es el punto real de partida del estudiantado y no aquel supuesto por el conjunto de docentes de las universidades públicas nacionales. La inclusión en términos de matrícula también obliga a una revisión de las prácticas docentes, para evitar asumir la homogeneidad de experiencias y trayectorias educativas que detentan quienes buscan acceder a la universidad. 
http://doi.org/10.15359/ree.24-3.27

http://www.una.ac.cr/educare

educare@una.ac.cr

\section{Las primeras generaciones en la UNLP}

En un relevamiento efectuado para atender características de estudiantes ingresantes de la UNLP, hallamos que pocos trabajos enfatizan en las dimensiones estructurales que permitieron el incremento de la matrícula universitaria. Tampoco fue posible encontrar alguna investigación que tratara sobre la totalidad de las facultades de la UNLP. Nuestra investigación se aloja en esta zona de vacancia, al mismo tiempo que busca constituirse en un escenario para futuros interrogantes que problematizan las políticas de inclusión desde la perspectiva de comunicación/educación.

En ese sentido, buscamos mostrar el incremento de la matrícula en la UNLP a partir de los datos recolectados por el CeSPI y una proyección histórica, entre el total acumulado desde 2008 hasta 2015.

Figura 1: Total acumulado de ingresantes UNLP por facultades (2008-2015)

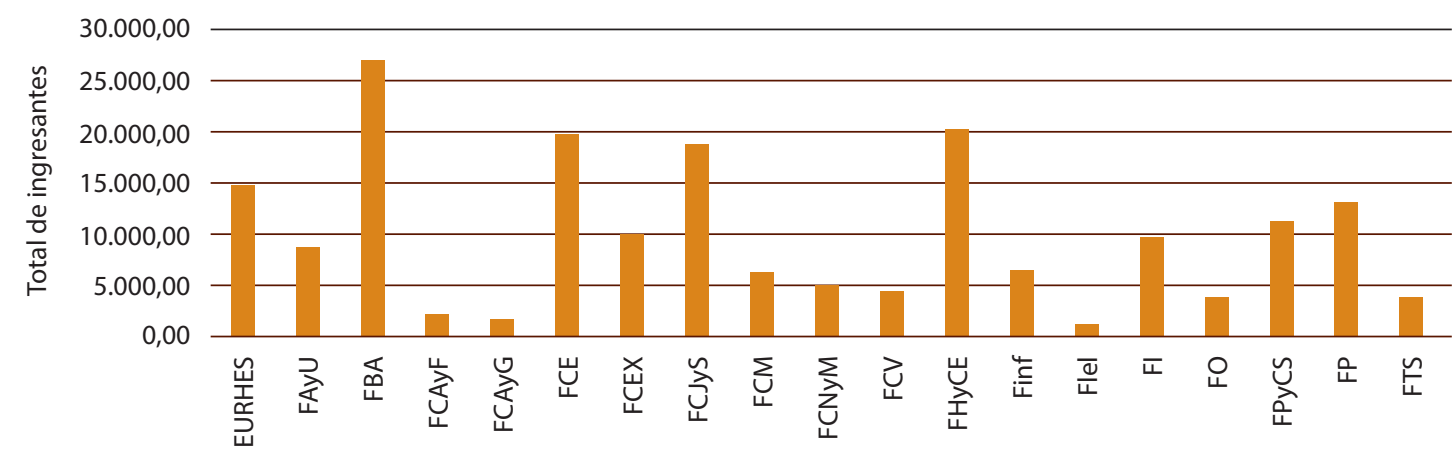

Nota: CeSPI. Elaboración propia.

Facultades de la UNPL

Figura 2: Total de ingresantes UNLP discriminados por año

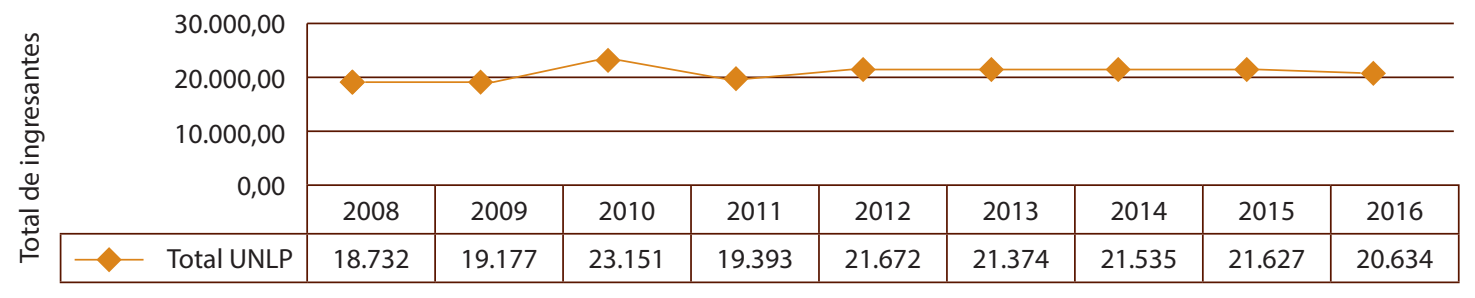

Nota: CeSPI. Elaboración propia.

Años 
http://doi.org/10.15359/ree.24-3.27

La evolución de la matrícula en la UNLP (Figura 1) ha tenido un crecimiento sostenido durante todo el periodo estudiado. En el total acumulado, se puede observar que el mayor número de ingresantes lo tiene la Facultad de Bellas Artes (24 732), seguida por la Facultad de Humanidades y Ciencias de la Educación (18 170), Facultad de Ciencias Económicas (17 958) y la Facultad de Ciencias Jurídicas y Sociales (16 887). El mayor pico se produjo en 2010, con una leve caída en el 2011; pero que también implica un mayor número de ingresantes respecto de 2009 (Figura 2). En este sentido, un aspecto a considerar es que la creación de nuevas universidades en el conurbano bonaerense (por ejemplo, la Universidad Nacional Arturo Jauretche -localizada en Florencio Varela - y la Universidad Nacional de Avellaneda para nombrar a las más próximas geográficamente a la ciudad de La Plata) no le quitó matrícula a la UNLP.

Para cruzarlo con otras tipos de variables económicas estructurales, este aumento de la matrícula también se originó cuando el ciclo de crecimiento de nuestro país había disminuido en su intensidad (Kessler, 2014) y también con el contexto de una crisis internacional acuciante como la que ocurrió en 2008 con la quiebra de Lehman Brothers. Aún en un escenario económico de crecimiento atenuado, el número de ingresantes fue aumentando, tal vez por la ayuda de políticas de inclusión educativa y otras de la misma tónica (programa de becas, asignación universal por hijo o hija, tarifas subsidiadas al transporte y servicios públicos, etc.). Una política económica contracíclica tiene sus efectos sobre las expectativas sociales relacionadas con el acceso, la permanencia y la acreditación de trayectorias educativas.

Figura 3: Total acumulado de ingresantes (2008-2015) discriminado por facultades y géneros masculino y femenino



Nota: CeSPI. Elaboración propia. 
http://doi.org/10.15359/ree.24-3.27

http://www.una.ac.cr/educare

educare@una.ac.cr

Figura 4: Total de ingresantes (2008-2015) discriminado por géneros masculino y femenino

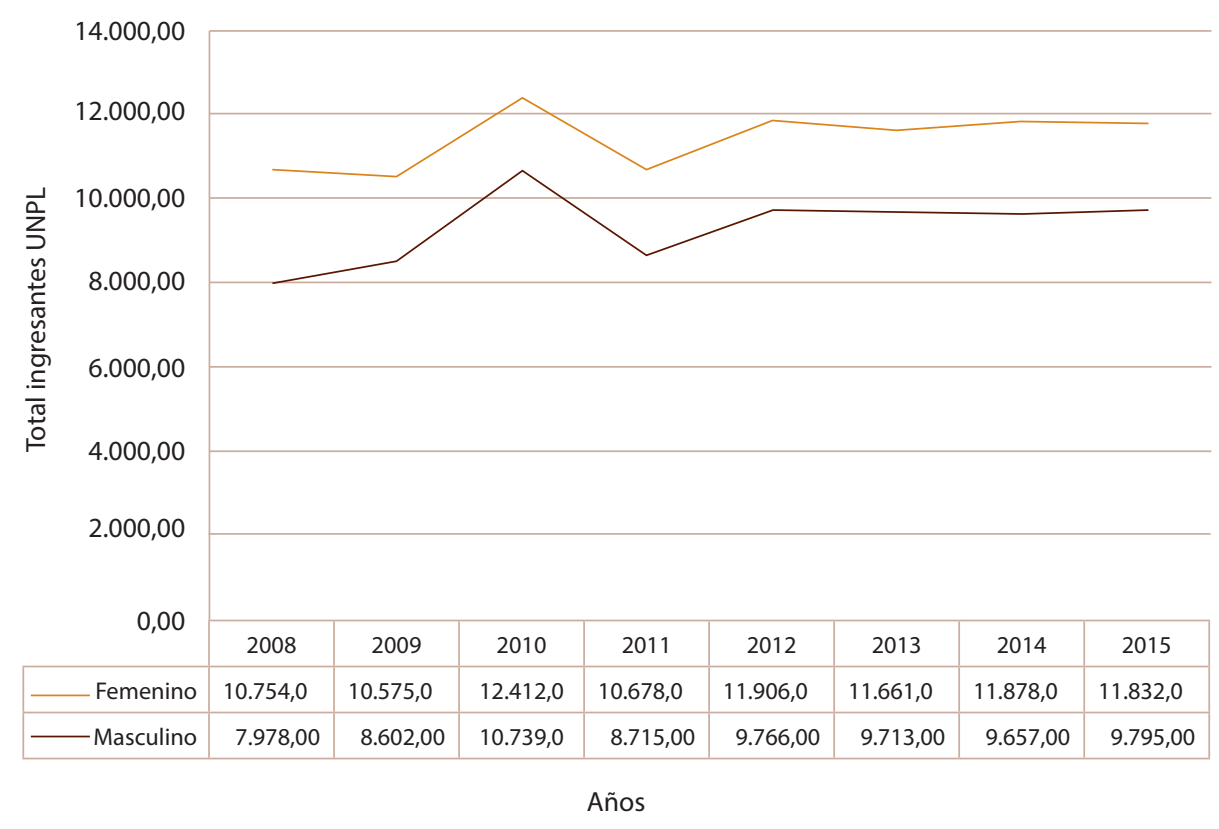

Nota: CeSPI. Elaboración propia.

La diferencia de géneros (Figuras 3 y 4) entre la totalidad de ingresantes también muestra una información reveladora. Las mujeres son mayoría en 12 facultades de la UNLP, además su crecimiento guardó el mismo patrón que la matrícula total del periodo analizado. Esta misma tendencia de mayor número de ingresantes mujeres se mantiene en todo el sistema universitario argentino. Así lo indica la Secretaría de Políticas Universitarias del Ministerio de Educación de la Nación (2011), al señalar que para el periodo 2011, en universidades nacionales y privadas, había un $56,6 \%$ de mujeres ingresantes contra el $43,4 \%$ de hombres. En la suma de la tasa de graduación de universidades de gestión pública y de gestión privada, las mujeres se gradúan en un 61,2 \% contra el 32,8 \% de hombres (Secretaría de Políticas Universitarias del Ministerio de Educación de la Nación, 2011). A pesar de un desarrollo desigual e inconexo de políticas de género, las mujeres han podido ingresar a sus estudios universitarios y graduarse en mayor número que los hombres. También revela los crecientes desafíos que deberán sostener las instituciones de educación superior para atender las problemáticas de género que se complejizan cada vez más y garantizar mejores condiciones de acceso, permanencia y acreditación de trayectorias formativas. 
http://doi.org/10.15359/ree.24-3.27

http://www.una.ac.cr/educare educare@una.ac.cr

Figura 5: Total de ingresantes UNLP y relación con primera generación de universitarios (2008-2015)

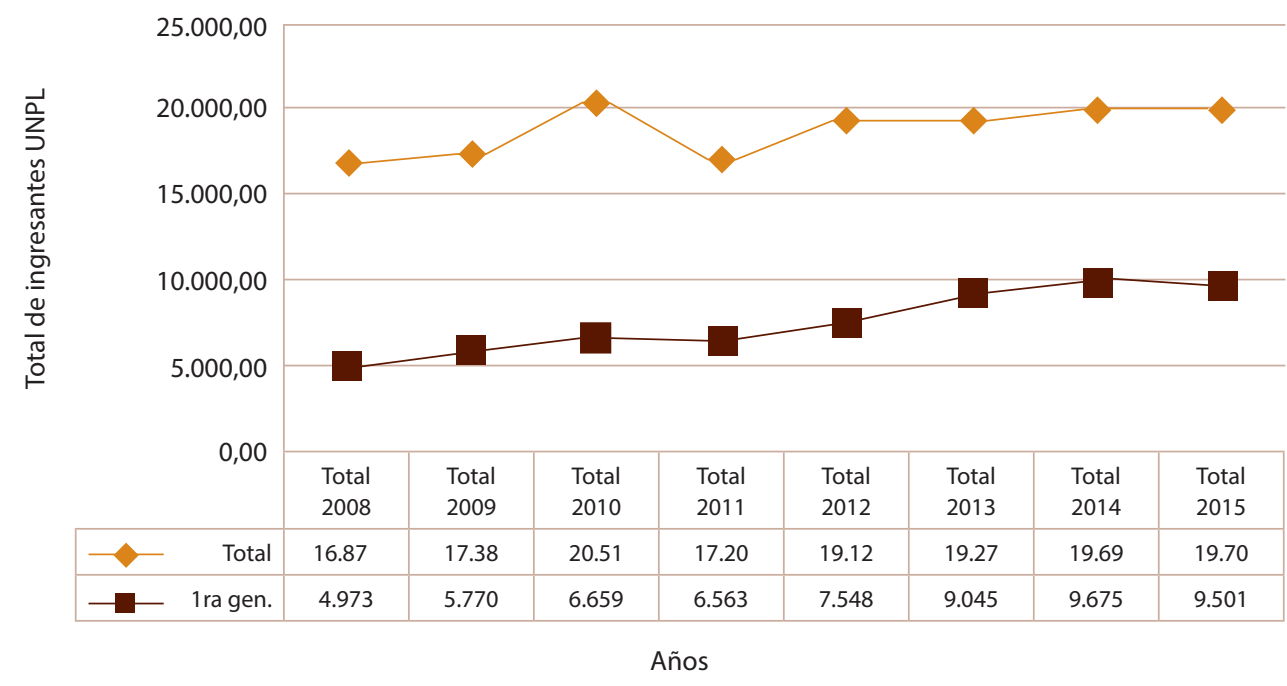

Nota: CeSPI. Elaboración propia. Aclaración: la Facultad de Ciencias Jurídicas y Sociales no informó al CeSPI sobre sus ingresantes primera generación de universitarios.

Figura 6: Porcentaje de ingresantes primera generación de universitarios respecto del total. Periodo 2008-2015

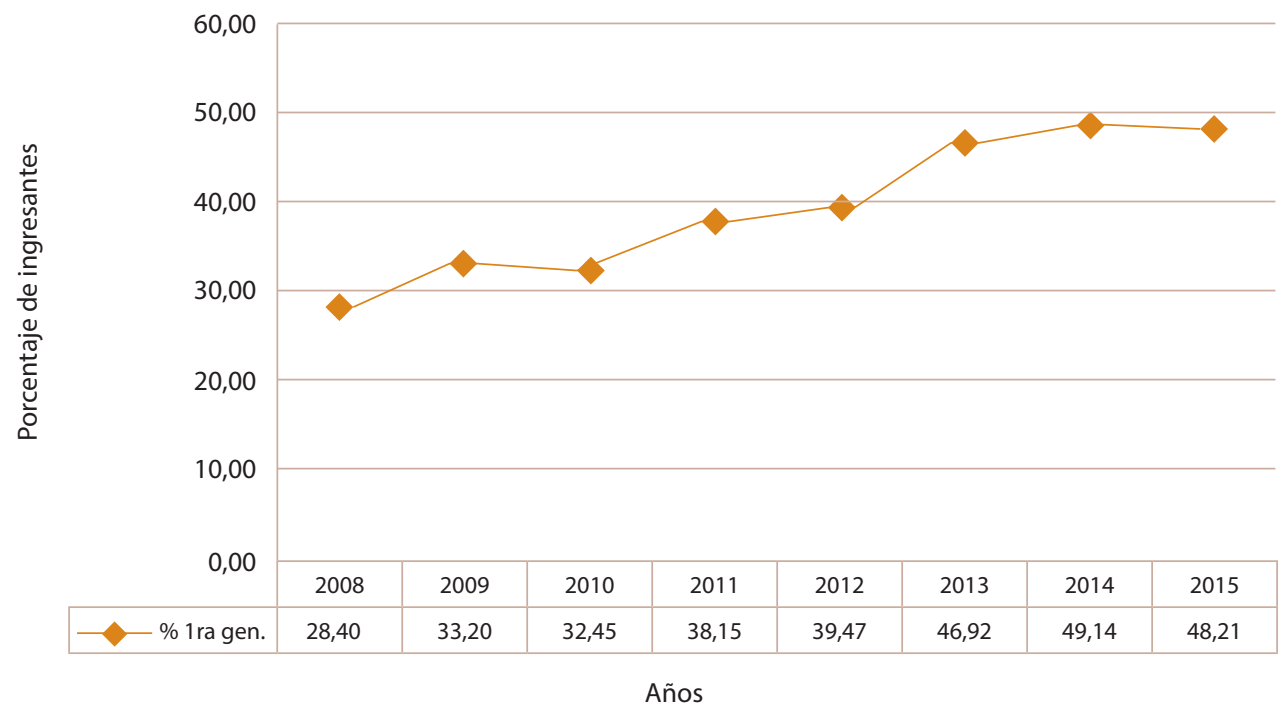

Nota: CeSPI. Elaboración propia. Aclaración: la Facultad de Ciencias Jurídicas y Sociales no informó al CeSPI sobre sus ingresantes primera generación de universitarios. 
http://doi.org/10.15359/ree.24-3.27

http://www.una.ac.cr/educare

educare@una.ac.cr

Las Figuras 5 y 6 muestran el crecimiento de ingresantes en primera en la primera generación. El incremento es sostenido, siempre fue en aumento con el correr de los años y sin descensos abruptos. Tal es así que la UNLP, una de las universidades públicas más importantes de la Argentina, llegó al bienio 2014-2015 con casi la mitad de sus ingresantes que forman parte de la primera generación universitaria. Como aclaramos al principio, se trata de estudiantes cuyos padres o madres no tienen títulos universitarios. Un aspecto también para resaltar consiste en que el momento de mayor ingreso total a la UNLP -año 2010- el porcentaje de primera generación no fue tan alto como podría suponerse del total absoluto. El aumento fue procesual, con un fuerte pico de crecimiento en 2013 (7,45 \%) respecto de 2012 y ese año representa el mayor porcentaje de variación entre un año y otro.

Esta dimensión de análisis sobre la primera generación universitaria de la UNLP casi no fue explorada en los trabajos relevados con anterioridad. Tampoco se encuentra información sistematizada en el Anuario 2011 de Estadísticas Universitarias de la SPU que describan el acceso de las primeras generaciones a los estudios superiores universitarios. Es posible encontrar numerosas afirmaciones que describen a las universidades localizadas en el conurbano bonaerense como instituciones compuestas en su mayoría por estudiantes de estas características (Colabella y Vargas, 2014; Tedesco, 2015).

Dentro de los interrogantes planteados en el inicio, también quisimos indagar en la cantidad de ingresantes a la UNLP que provenían del Plan Fines. Para contextualizar, se trata de un plan de terminalidad educativa inscripta en el artículo 138 de la Ley de Educación Nacional, cuyo objetivo era garantizar la finalización de la escolaridad a jóvenes y personas adultas que no hubieran iniciado o completado la secundaria. La magnitud de esta iniciativa del Estado es de una importancia supina y todavía no fue estudiada con la suficiente exhaustividad para analizar su impacto cuantitativo y cualitativo en todo el territorio nacional. Solo en la provincia de Buenos Aires, donde se encuentra alojada geográficamente la UNLP, en el año 2010 había alrededor de 58 mil estudiantes, mientras que en el 2012 se llegó a una cifra cercana a los 100 mil en condición de adultez; en 2015, con el incentivo que significaron las políticas para jóvenes como el PRO.GRE. SAR y Plan Jóvenes; se estima que 250 mil personas adultas cursaron en esa modalidad en el Plan Fines. Hasta el año 2015, se titularon 20 mil estudiantes en toda la provincia de Buenos Aires, de los cuales 3200 corresponden al distrito de la ciudad de La Plata (Mañez, 2015). Señalamos la cuestión de la titulación porque nuestra investigación nos indica que en la provincia de Buenos Aires y la ciudad de La Plata es considerable el número de estudiantes que culminaron sus trayectorias educativas y aprobaron todas sus materias; pero carecen del certificado del Estado que lo acredite. La excesiva burocratización de las dependencias estatales, la ausencia de una centralización ágil y los insuficientes esfuerzos de la coordinación interministerial de las jurisdicciones implicadas es la explicación más contundente para este retraso. 
http://doi.org/10.15359/ree.24-3.27

La Tabla 1 muestra la cantidad de ingresantes provenientes del Fines a la UNLP por cada una de sus facultades y en qué años se produjeron esos ingresos.

Tabla 1: Ingresantes de Fines a la UNLP

\begin{tabular}{|c|c|c|c|}
\hline Facultad & 2012 & 2015 & 2016 \\
\hline Escuela Universitaria de Recursos Humanos del Equipo de Salud & & 9 & 19 \\
\hline Facultad de Arquitectura y Urbanismo & & & 1 \\
\hline Facultad de Bellas Artes & 1 & 3 & 6 \\
\hline Facultad de Ciencias Astronómicas y Geofísicas & & & 1 \\
\hline Facultad de Ciencias Exactas & & & 2 \\
\hline Facultad de Ciencias Medicas & & & 6 \\
\hline Facultad de Humanidades y Ciencias de la Educación & & & 4 \\
\hline Facultad de Informática & & & 4 \\
\hline Facultad de Informática e Ingeniería & & & 2 \\
\hline Facultad de Ingeniería & & & 2 \\
\hline Facultad de Periodismo y Comunicación Social & & & 5 \\
\hline Facultad de Psicología & & & 4 \\
\hline Facultad de Trabajo Social & & & 6 \\
\hline
\end{tabular}

Nota: CeSPI. Elaboración propia.

La relación entre la cantidad de ingresantes provenientes del Plan Fines y el total de la UNLP es ínfima. Es decir, no hubo un incremento significativo de estudiantes que finalizaron su educación secundaria en un programa de terminalidad y su correspondiente ingreso a una institución de educación superior universitaria. Tampoco afirmamos que la acreditación de la educación secundaria deba corresponderse exclusivamente con la continuidad universitaria, más bien señalamos que, dada la magnitud cuantitativa del Fines, hay una escasa proporción de estudiantes que se hayan figurado como un horizonte de expectativas ingresar a la UNLP. Al mismo tiempo señala un trayecto de posible abordaje institucional que deban contemplar las universidades para organizar políticas de acercamiento, ya no a escuelas secundarias, sino también al complejo y heterogéneo conglomerado de organizaciones sociales que tienen sedes para el dictado de clases en el marco del plan Fines. 
http://doi.org/10.15359/ree.24-3.27

http://www.una.ac.cr/educare

educare@una.ac.cr

\section{Conclusiones}

Las políticas inclusivas de educación son materia de debates constantes, confrontaciones político-electorales, reforzamiento del sentido común mediático, entre otras. A veces, representan todo esto y mucho más. Con el objetivo de aportar al diálogo, buscamos focalizar en el ingreso en periodo 2008-2015 a la UNLP. Por ejemplo, el total de ingresos y su proyección temporal, como también la cuestión de los ingresantes primera generación. El conjunto de materiales abordados que analizaban cuestiones similares en nuestra universidad parecían darnos la razón acerca de nuestras preocupaciones. Una primera cuestión a atender consiste en que es infructuoso mencionar la existencia de políticas inclusivas en educación sin la interrelación con otras políticas que confluyan en ese mismo horizonte estratégico. En reiteradas oportunidades, estas incoherencias se le asignan al sistema público de enseñanza para que actúe como el suturador del conflicto social. Si ni la política, ni la economía incluyen a los sectores sociales más relegados, la escuela sola jamás podrá hacerlo. El sentido común (mediático) suele pedir que las políticas públicas de educación sean inclusivas, igualitarias, mientras que, al mismo tiempo, avala y legitima políticas económicas de endeudamiento, especulación financiera y de profunda desconfianza por lo público.

Brindar algunas pistas de los elementos contextuales permitió inscribir las políticas públicas de educación con otras cuyos intereses se centraban en diversos escenarios. De allí que efectuamos un breve recorrido por algunos elementos estructurales de la sociedad argentina en los últimos años. Coincidimos con Kessler (2014) en que la sociedad argentina actual es menos desigual, respecto de los años noventa, porque las tendencias generales son contrapuestas a los de dicha década. Pero, además, es menos desigual porque la demanda de igualdad está omnipresente en el lenguaje de las reivindicaciones y en la forma en que se miran y evalúan distintas situaciones políticas. Sin embargo, consideramos que en un análisis de este tipo también deben sumarse interrogantes acerca de la conformación del sistema de medios de comunicación -públicos y privados- de nuestro país. Porque, si bien el significante igualdad siempre está dentro de un terreno de disputas y es una fuente inagotable de luchas por derechos, en algunas de sus dimensiones se encuentra modelado para ciertos sectores sociales, a partir de las formaciones discursivas de los medios.

El acceso al sistema de enseñanza universitaria también puede ser inscripto en los debates sobre la desigualdad. Desde allí es que buscamos aportar con nuestro análisis, pero realizando algunas aclaraciones específicas. La igualdad es una demanda que pueden realizar diversos colectivos sociales, organizaciones políticas, institucionalidades en proceso de formación o ya consolidadas, pero solo el Estado es quien debe y puede garantizar la inclusión. Consideramos la inclusión como la posibilidad de acceso a una institucionalidad educativa que aparecía vedada para las experiencias históricas de algunos sectores sociales y también como la oportunidad de peticionar por el derecho a ingresar a la educación universitaria. Cuando acontece un escenario de inclusión real por parte del Estado, las subjetividades implicadas en esas políticas ensanchan sus horizontes de experiencias y complejizan cada vez más las reivindicaciones por sus derechos. 
La información develada demuestra la alta tasa de escolarización de todos los niveles sociales que tiene la Argentina respecto de otros países latinoamericanos. Dentro de esa dinámica se encuentra el aumento de la matrícula de ingresantes a la UNLP, que fue procesual y a la vez demuestra el fruto de los resultados de otras políticas públicas, no solo las educativas. La mejor distribución del ingreso y las medidas económicas contracíclicas tuvieron incidencia en esta problemática. En el periodo 2008-2015, en la UNLP hubo una porción significativa de la sociedad que se figuró la universidad pública como una expectativa de futuro. En el año 2014 y 2015, casi la mitad de quienes buscan acceder a sus estudios universitarios en la UNLP forman parte de ese colectivo. Acceder a una nueva institucionalidad educativa tiene incidencias formativas no solo en el estudiantado universitario, sino también en su entorno cotidiano más inmediato. Desde comunicación/ educación, sabemos que en este proceso se despliegan un juego de interpelaciones y reconocimientos que generan nuevas lecturas y reescrituras del mundo.

También es importante analizar lo que permanece en lo que cambia y ver qué es lo que se modifica en lo que perdura, según lo conciben los estudios culturales. La cantidad de ingresantes que provienen del Plan Fines es ínfima respecto del total absoluto de ingresantes a la UNLP. Esto indica que todavía, para un sector importante de la sociedad, la reparación del derecho a la educación secundaria no se traduce en una expectativa de continuar sus estudios universitarios.; al menos que se lo puedan figurar como una posibilidad. Sin embargo, atendemos que todavía operan condicionamientos estructurales que obturan las construcciones de esas trayectorias para un universo que tuvo un recorrido particular dentro de las instituciones educativas del sistema público de enseñanza. Un ciclo político puede contribuir a modificar rasgos de la estructura social, pero es probable que muchos de sus esfuerzos sean tenues respecto de las diferentes instancias de apropiación desigual de los capitales culturales. Hubo mejoras importantes, pero insuficientes, en términos temporales y en intensidad política, en atender algunas dimensiones problemáticas para reducir la desigualdad.

En síntesis, las políticas educativas inclusivas tienen que acontecer de manera articulada con otras políticas públicas que tengan el mismo horizonte estratégico. De nada sirve pedirle al sistema educativo que incluya a los sectores sociales postergados cuando el resto de las políticas estatales no lo hace. En nuestro caso, vimos cómo, a partir de eso, el Estado acercó a un número considerable de estudiantes para que pudieran representarse el sistema universitario como una expectativa de futuro. Tampoco es descabellado considerar que, en un tiempo futuro, el acceso a los estudios universitarios se transforme en una demanda activada por sectores políticos diversos. Para la UNLP, que casi la mitad de sus ingresantes sean primera generación universitaria modifica completamente su panorama, la obligará a encontrar mecanismos institucionales para atender a estas nuevas subjetividades. Veremos hasta qué punto es posible, en un nuevo contexto político de signo inverso y que parecería apuntar a intensificar la desigualdad. 
http://doi.org/10.15359/ree.24-3.27

http://www.una.ac.cr/educare

educare@una.ac.cr

\section{Declaración de Material complementario}

Este artículo tiene disponible como material complementario:

- La versión preprint del artículo en https://doi.org/10.5281/zenodo.3341826

\section{Referencias}

Aberbuj, C. y Zacarías, I. (2015). La universidad y los desafíos de la pedagogía. En J. C., Tedesco (Ed.), La educación argentina hoy. La urgencia del largo plazo (pp. 351-382). Veintiuno Editores.

Arceo, N., Barrera, M., González, M. y Manzanelli, P. (2016). Informe de coyuntura n. ${ }^{\circ} 21$. Noviembre de 2016. CIFRA- CTA. http://www.centrocifra.org.ar/docs/IC\%2021.pdf

Basualdo, E. M. (2005). Estudios de historia económica argentina. Desde mediados del siglo XX a la actualidad. Siglo Veintiuno Editores.

Basualdo, E., Barrera, M. A. y Manzanelli, P. (2015). La naturaleza política y la trayectoria económica de los gobiernos kirchneristas. CIFRA-CTA. https://www.slideshare.net/IADERE/ la-naturaleza-poltica-y-la-trayectoria-econmica-de-los-gobiernos-kirchneristas

Benza, G. (2016). La estructura de clases argentina durante la década 2003-2013. En G. Kessler (Ed.), La sociedad argentina hoy. Radiografía de una nueva estructura (pp.111-140). Siglo Veintiuno Editores.

Bourdieu, P. (2005). Capital cultural, escuela y espacio social. Siglo XXI.

Colabella, L. y Vargas, P. (2014). "La Jauretche". Una universidad popular en la trama del sur del Gran Buenos Aires. En N. Gluz, M. Karolinski, I. Rodríguez Moyano, C. Talavera, I. Gaona López, P. V. Gadelha Mendes, L. Colabella y P. Vargas (AA.), Avances y desafíos en políticas públicas educativas. Análisis de casos en Argentina, Brasil, Colombia y Paraguay (pp.251321). Clacso. https://www.academia.edu/11587187/Avances y desaf\%C3\%ADos en Pol\%C3\%ADticas P\%C3\%BAblicas Educativas. An\%C3\%A1lisis de casos en Argentina Brasil Colombia y Paraguay

de la Fare, M. (2010). Principales ideas, discusiones y producciones en educación de jóvenes y adultos en Argentina. Aportes para una reconstrucción histórica. Ministerio de Educación de la Nación; DINIECE.

Ezcurra, A. M. (2011). La igualdad en educación superior. Un desafío mundial. Universidad Nacional de General Sarmiento. https://www.academia.edu/40234752/lgualdad en educaci\%C3\%B3n superior 
http://doi.org/10.15359/ree.24-3.27

Gentili, P. (2015). América Latina, entre la desigualdad y la esperanza. Crónicas sobre educación, infancia y discriminación. Siglo Veintiuno Editores.

Gessaghi, V. (2016). La educación de la clase alta argentina. Entre la herencia y el mérito. Siglo Veintiuno Editores.

Heredia, M. (2016). Las clases altas y la experiencia del mercado. En G. Kessler (Comp.), La sociedad argentina hoy. Radiografía de una nueva estructura (pp. 185-205). Siglo Veintiuno Editores.

Kessler, G. (2014). Controversias sobre la desigualdad. Argentina, 2003-2013. Fondo de Cultura Económica.

Kessler, G. (2016). La sociedad argentina hoy. Radiografía de una nueva estructura. Siglo Veintiuno Editores.

Mañez, N. (2015). Prácticas comunitarias y educativas a partir del PLAN FINES: experiencias de centros comunitarios del gran La Plata [Tesis de grado]. Universidad Nacional de La Plata, Argentina.

Martínez, D. G. (2015). Saberes, experiencias y subjetividades de la educación de jóvenes y adultos en la ciudad de La Plata. Un abordaje desde la perspectiva de comunicación/educación [Tesis doctoral inédita] Universidad Nacional de La Plata, Argentina. http://sedici.unlp.edu.ar/ handle/10915/49538

Morabes, P., Martínez, D. G. y Vissani, V. (2017). Articulaciones estratégicas en comunicación/ educación para leer a las políticas públicas inclusivas en la educación de jóvenes y adultos en Argentina (2003-2015). Revista Latinoamericana de Ciencias de la Comunicación, 14(26), 164-175. http://sedici.unlp.edu.ar/handle/10915/72221

Reygadas, L. (2008). La apropiación. Destejiendo las redes de la desigualdad. Anthropos.

Ruta, C. (2015). El futuro de la universidad argentina. En J. Tedesco (Ed.), La educación argentina hoy. La urgencia del largo plazo (pp. 319- 350). Siglo Veintiuno Editores.

Secretaría de Políticas Universitarias del Ministerio de Educación de la Nación. (2011). Anuario 2011 estadísticas universitarias. Ministerio de Educación de la República Argentina.

Sivak, M. (2013). Clarín, el gran diario argentino. Una historia. Planeta.

Tedesco, J. (Comp.). (2015). La educación argentina hoy. La urgencia del largo plazo. Siglo Veintiuno Editores.

Unesco (2017). Perfiles de país: Argentina. SITEAL. http://www.publicaciones.siteal.iipe.unesco. org/perfiles-de-pais/4/argentina 